\title{
Numerically Investigating the cause of Broadband Lasing from InAs/InP Quantum-dash Laser
}

\author{
M. Z. M. Khan, Tien K. Ng, and Boon S. Ooi* \\ Photonics Laboratory, Computer, Electrical, and Mathematical Sciences and Engineering (CEMSE) Division, \\ King Abdullah University of Science \& Technology (KAUST), Thuwal 23955-6900, Kingdom of Saudi Arabia (KSA) \\ *corresponding author: boon.ooi@kaust.edu.
}

\begin{abstract}
The authors numerically investigated the origin of broadband lasing from multi-stack InAs/InP quantum dash (Qdash) laser. For this a model based on multi-population carrier-photon rate equation is developed which treats each Qdash stacking layer separately. In addition, the coupling between the adjacent stacks is also accounted in the model apart from the inhomogeneous broadening due to dash size or composition fluctuation. Simulation results show that the effect of Qdash inhomogeneity on a single plane (in-plane or localized inhomogeneity) is negligible in broadening the lasing spectra compared to the inhomogeneity across the stacks that are emitting at different wavelength. Our results would help in further optimizing the wafer structure design and improve the lasing bandwidth.
\end{abstract}

Key words - Quantum dash laser; rate equation model; broadband lasers; chirped barrier;

\section{INTRODUCTION}

The inhomogeneous broadening due to size or composition dispersion in the multi-stack quantum dots (Qdots)/ Qdashes nano-structures active region, grown by self assembled growth technology, is undesirable because of resultant inferior device performance. Nonetheless, the inherent inhomogeneous broadening from the self-assemble growth technology have been exploited recently to realize what is called broadband laser diodes (LDs) [1-4] and superluminescent diodes (SLDs) [5-7]. These devices span emission bandwidth as large as $\sim 42 \mathrm{~nm}(>250 \mathrm{~nm})$ attaining power in few hundreds (tens) of $m W$ from broadband LDs (SLDs). These class of near-infrared broadband emitters find vast application in multi-disciplinary field of application viz. gas sensing, medical imaging, diagnostic systems, spectroscopy, optical communications, etc $[1,2]$. Therefore, it becomes important to understand the effect of inhomogeneous broadening in the multi-planar epitaxial structures, which are typically used to increase the device performance. Recently, through optical characterization, it was shown implicitly that the variation of inhomogeneous broadening across the multi-stack is a significant factor in increasing the overall inhomogeneity of the system and that the inhomogeneous broadening in the single plane is trivial [8]. This is an important observation that requires further analysis since it would help in further optimization of wafer structure design for broadband emitter realization.

The objective of this paper is to numerically show in an explicit manner that indeed the inhomogeneous broadening across the stacks defines the overall inhomogeneity of the multi-stack device epitaxial structure by considering a broadband InAs/InP Qdash laser structure. For this, we have developed a rate-equation model that takes into account the carrier-photon dynamics in each of the energy level. In addition, the model treats each stacking layers and the respective inhomogeneous broadening individually, along with the carrier coupling effect among the Qdash layers. This includes carrier diffusion and tunneling through the barrier layers.

\section{NUMERICAL MODEL}

The model is based on carrier and photon dynamics represented by rate equations and incorporate the coupling among the adjacent Qdash stacks. The reservoir of carriers is the separate-confined heterostructure followed by the wetting layer and the dash ground state energy level (we assumed only ground state energy level in dashes). The associated time constants are $\tau_{S W}$ (relaxation from separate-confined heterostructure to wetting layer), $\tau_{W D}$ (relaxation from wetting layer to Qdash ground state), $\tau_{D W}$ (re-excitation from Qdash ground state to wetting layer), $\tau_{W S}$ (re-excitation from wetting layer to separate-confined heterostructure ), and $\tau_{S}$, $\tau_{W}, \tau_{D}$ corresponding to the recombination in separateconfined heterostructure, wetting layer and Qdash ground state, respectively. More details can be found elsewhere [911]., and only electron dynamics are considered in the formulation with the hypothesis that the holes being faster follows the electrons. We have considered $n=1,2, \ldots N_{l y r}$ stacking layers. $N_{S}$ and $N_{W}$ refers to the total number of carriers in the separate-confined heterostructure and wetting layer, while $N_{j, k}$ represents the carrier dynamics in each of the intra-dash energy levels of dash group. The subscripts $j$ and $k$ refers to the $j^{\text {th }}$ group of Qdash ensemble and its $k^{\text {th }}$ intra-dash energy level. The rate equations of the carrier population of these energy level are formulated in a similar manner following reference [12]. The rate equation of the separate-confined heterostructure layer is:

$$
\frac{d N_{S}}{d t}=\frac{I}{e}-\frac{N_{S}}{\tau_{S W}}-\frac{N_{S}}{\tau_{S}}+\frac{N_{W}^{N_{\text {lyr }}}}{\tau_{W S}}
$$

where the first term on the right hand side of Eqn. (1) correspond to the number of carriers injected into the separate-confined heterostructure layer, and $\tau_{S}$ is the non radiative recombination time of the separate-confined heterostructure layer. Similarly, rate equations in the wetting layer and Qdash GS energy level is formulated taking into consideration the coupling among the stacks. These equations are then coupled with the multimode photon rate equation:

$$
\frac{d S_{m}}{d t}=\beta \sum_{n, j, k} B\left(E_{m}-E_{j, k}^{n}\right) \frac{N_{j, k}^{n}}{\tau_{S p}}+\frac{c}{n_{a}} \sum_{n, j, k} \Gamma^{n} g_{n, m}^{j, k} S_{m}-\frac{S_{m}}{\tau_{p}}
$$


TABLE I

PARAMETERS USED IN THE SIMULATION

\begin{tabular}{|c|c|c|c|}
\hline Parameter & Description & Value & Unit \\
\hline$L$ & Cavity length & 2000 & $\mu m$ \\
\hline$d$ & Stripe width & 3 & $\mu m$ \\
\hline$w_{W L}$ & Wetting layer thickness & $(1,1,1,1)$ & $n m$ \\
\hline$w_{D H}$ & QDash width & 20 & $n m$ \\
\hline$h_{D H}$ & QDash height & $(3.2,2.9,2.6,2.3)$ & $n m$ \\
\hline$N_{h r}$ & Number of QDash layers & 4 & - \\
\hline$A_{e f f}$ & QDash effective crossection area & $(8.0,7.2,6.5,5.7) \times 1 \mathrm{e}-11$ & $\mathrm{~cm}^{2}$ \\
\hline$\Gamma$ & Confinement factor & $(0.01,0.01,0.01,0.01)$ & - \\
\hline$R_{1}=R_{2}$ & Cleaved facet reflectivity & 0.3 & - \\
\hline$\alpha_{i}$ & Internal modal loss & 7.5 & $\mathrm{~cm}^{1}$ \\
\hline$\beta$ & Spontaneous emission factor & $1 \mathrm{e}-4$ & - \\
\hline$N_{D}$ & QDash density of states & $1 \mathrm{e} 18$ & $\mathrm{~cm}^{-3}$ \\
\hline$D_{g}$ & QDash ground state degeneracy & 1 & - \\
\hline$D_{W}$ & WL density of states & $4.24 \mathrm{e} 18$ & $\mathrm{~cm}^{3}$ \\
\hline$E_{C V}$ & Central transition energy & $(743,763,783,803)$ & $\mathrm{meV}$ \\
\hline$E_{W L}$ & WL ground state energy & 890 & $\mathrm{meV}$ \\
\hline$\hbar \Gamma_{h o m}$ & Homogeneous broadening & 10 & $\mathrm{meV}$ \\
\hline$\tau_{s \pi}$ & Relaxation time from $\mathrm{SCH}$ to $\mathrm{WL}$ & 500 & ps \\
\hline$\tau_{\text {TS }}$ & $\begin{array}{l}\text { Re-excitation time from WL to } \\
\text { SCH }\end{array}$ & 1 & $n s$ \\
\hline$\tau_{W D O}$ & $\begin{array}{l}\text { Initial capture time from WL to } \\
\text { QDash ground state }\end{array}$ & 2 & ps \\
\hline$\tau_{\pi}$ & Recombination lifetime of WL & 0.8 & ns \\
\hline$\tau_{D}$ & Recombination lifetime of QDash & 0.5 & $n s$ \\
\hline$\tau_{S p}$ & Spontaneous lifetime & 2.8 & ns \\
\hline$\tau_{p}$ & Photon lifetime & 3.4 & ps \\
\hline$n_{a}$ & Refractive index & 3.5 & - \\
\hline
\end{tabular}

Eqn. (2) includes the spontaneous emission term, stimulated emission term and the photon loss where the photon population $S_{m}$ is proportional to the linear gain $g_{n, m}^{j, k}$ which is calculated as the sum of the contributions of the various states at the $m^{\text {th }}$ mode recombination energy $E_{m}$. The details of various terms in Eqn. 2 and other carrier rate equations are reported in literature [9]. In brief, $g_{n . m}{ }^{j, k}$ incorporates both, the Lorentzian homogeneous broadening $B\left(E_{m}-E_{j, k}^{n}\right)$ and the Gaussian inhomogeneous broadening, $G_{j, k}^{n}$, with full width at half maximum (FWHM) $\Gamma_{i n h}$. Note that the volumetric density of states of the Qdashes, $N_{D}$, and $G_{j, k}^{n}$ includes the unique features of the dashes. The carrier relaxation and the re-excitation rates from wetting layer to Qdash energy states are calculated according to [10] by considering the initial relaxation time $\tau_{W D O}$ (capture time when the $k^{\text {th }}$ state $j^{\text {th }}$ Qdash group is unoccupied). The model is solved in the time domain by fourth order Runge-Kutta method by applying an input step current at $t=0$. Subsequently, the laser output power of the $m^{\text {th }}$ mode photon of energy $\hbar \omega_{m}$ from one cavity facet is obtained by:

$I_{m}=\hbar \omega_{m} c S_{m} \ln (1 / R) /\left(2 L n_{a}\right)$

where $R$ is the reflectivity of one cavity facet, $n_{a}$ is the refractive index of the active region taken as $3.5, L$ is the cavity length and $c$ is the speed of light. More details can be found in ref [10].

\section{RESULTS AND DISCUSSION}

We have considered four stack InAs/InP Qdash laser structure with varying barrier thickness $(10,15,20 \mathrm{~nm})$. More details of the structure and the simulation parameters utilized in this work are taken from $[4,9,10]$ and are shown in Table. 1. The room temperature calculated lasing spectra at different inhomogeneous broadenings, with increasing current injection, are shown in Figs. 1 and 2. In general, an overall
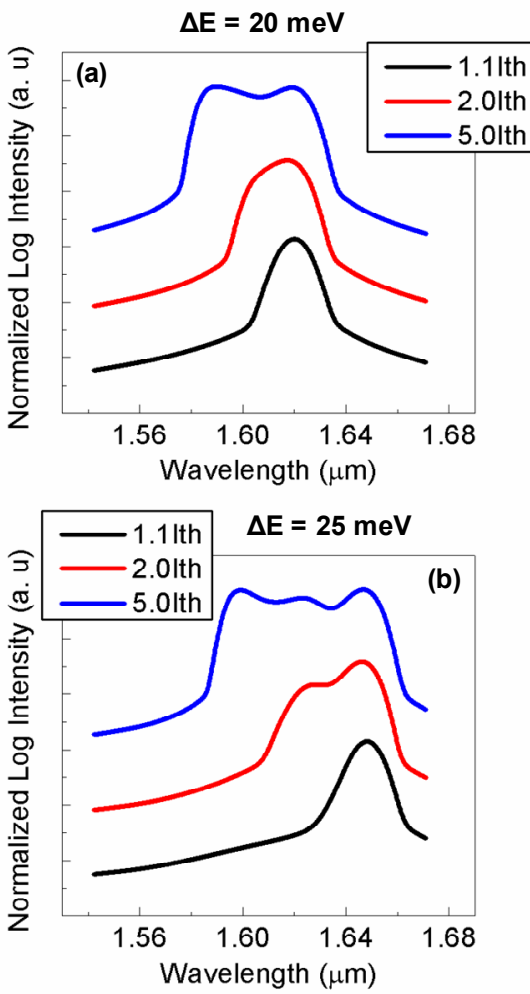

Fig. 1. Calculated progressive lasing spectra of the Qdash laser structure with 10 - 15 - $20 \mathrm{~nm}$ barriers, at different current injection. The in-plane inhomogeneous broadening is fixed at $\Gamma_{i n h}=(30,30,25,25) \mathrm{meV}$, while the inhomogeneity across the stacks is varied as (a) $\Delta E=20 \mathrm{meV}$, and (b) $\Delta E=$ $25 \mathrm{meV}$. The remaining parameters are unaltered.

inhomogeneous broadening of an active region (caused by either dash size variation, particularly height, or composition, or both) is characterized by the in-plane (or localized) inhomogeneity of a single plane (or layer) $\Gamma_{i n h}$, and the inhomogeneity across the dash layers $\Delta E$ (i.e. the difference in peak emission energies of adjacent Qdash stacks). In order to have a comprehensive understanding of effect of both the types of inhomogeneous broadening on the lasing spectra of Qdash lasers, we first fixed the in-plane inhomogeneous broadening term $\Gamma_{i n h}=(30,30,25,25) \mathrm{meV}$ while the inhomogeneous broadening across the stacks, $\Delta E$ is varied from $20 \mathrm{meV}$ to $25 \mathrm{meV}$, respectively. The corresponding lasing spectra are shown in Fig. 1(a) and (b). A progressive increase in the lasing bandwidth (calculated at FWHM) with increasing current is evident from the figures. This is attributed to the initial lasing from dash groups with intermediate average height (since height basically defines the GS energy level) once the total device loss is compensated. A substantial increase in the lasing bandwidth is observed on increasing $\Delta E$. The lasing FWHM in Fig. 1(a) and (b) attains a value of $\sim 43 \mathrm{~nm}$ and $\sim 61 \mathrm{~nm}$, respectively, at $5 \mathrm{I}_{\text {th }}$, which is an increase by $\sim 30 \%$. This is attributed to the availability of highly inhomogeneous dash density of states with wide span of energy transitions among quantized states. The short wavelength broadening is contributions from the smaller height dash groups, as a result of band filling effect. The broadening at the longer wavelength indicates that the larger height dash groups also starts lasing once they reach the condition of stimulated emission. Note that there is always competition between the small and large dash groups since the photon re-absorption process cannot be ignored, but, 

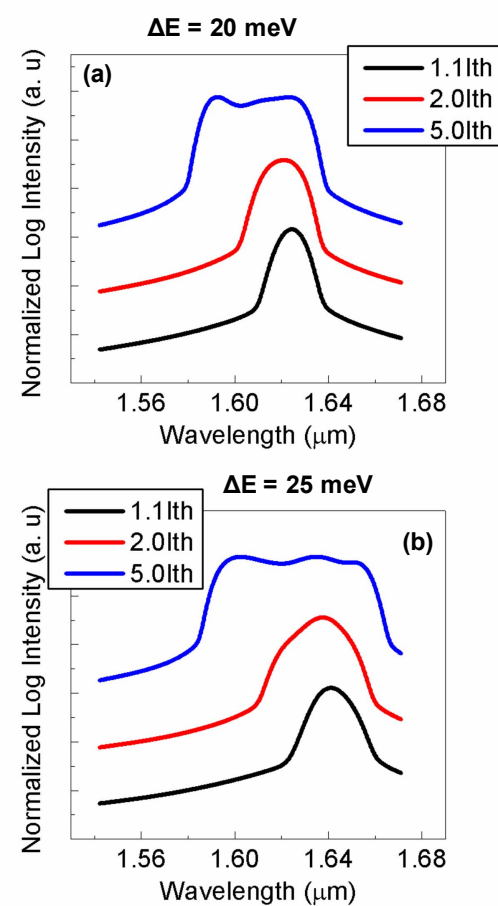

Fig. 2. Calculated progressive lasing spectra of the Qdash laser structure with $10-15-20 \mathrm{~nm}$ barriers, at different current injection. The in-plane inhomogeneous broadening is fixed at $\Gamma_{i n h}=(35,35,30,30) \mathrm{meV}$, while the inhomogeneity across the stacks is varied as (a) $\Delta E=20 \mathrm{meV}$, and (b) $\Delta E=$ $25 \mathrm{meV}$. The remaining parameters are unaltered.

with increasing injection, it is possible for both the groups to lase concurrently, thus enhancing the lasing spectra. Furthermore, the broadening of the lasing spectra at both short and long wavelength is due exclusively to the radiative recombination within Qdash GS and discards the possibility of device heating since the model does not take into account temperature dependent Qdash energy transitions.

The effect of increasing in-plane inhomogeneous broadening $\Gamma_{i n h}$ by $5 \mathrm{meV}$ in each of the Qdash stacks is depicted in Fig. 2 at two different values of $\Delta E$ (20 and 25 $m e V)$. A similar progressive increase in the lasing FWHM is seen on increasing current injection in both the figures. Considering Fig. 2(a) with $\Gamma_{\text {inh }}=(35,35,30,30) \mathrm{meV}$ and $\Delta E$ $=20 \mathrm{meV}$, a negligible increase in the lasing FWHM is observed on increasing $\Gamma_{i n h}$ compared to that of Fig. 1(a) which correspond to less in-plane inhomogeneous broadening system $\left(\Gamma_{i n h}=(30,30,25,25) \mathrm{meV}\right)$. The calculated lasing bandwidth in this case is $\sim 45 \mathrm{~nm}$ which is similar in value as obtained in Fig. 1(a). Thus our results further affirms the experimental results reported in ref [8] that the inhomogeneity across the stacks $(\Delta E)$ is a significant contributor to the overall inhomogeneous broadening of the active region. We postulate that the in-plane dispersion of the dash GS energy transitions is masked by the overall dispersion of dash GS energy transition across the entire active region (across all the stacks). We also plotted the progressive lasing spectra at $\Gamma_{i n h}=(35,35,30,30) \mathrm{meV}$ with $\Delta E=25 \mathrm{meV}$ in Fig. 2(b). Again, no appreciable increase in the lasing bandwidth is observed on increasing the $\Gamma_{i n h}$ value. The calculated lasing bandwidth in this case $(\sim 65 \mathrm{~nm})$ is comparable to Fig. 1(b) $(\sim 61 \mathrm{~nm})$ thus confirming that proper bandgap engineering of the Qdash stacks could lead to enhanced broadening of the lasing spectra.

\section{CONCLUSION}

In conclusion, we have developed a rate equation model for the Qdash lasers taking into account each dash layer individually, and the coupling between adjacent Qdash stack. We numerically showed that the inhomogeneity across the multi-stack Qdash laser structure is responsible for broadening of the lasing spectra rather than in-plane inhomogeneity. This analysis sheds light on the origin of broadband emission from the laser device and thus pave path to the optimization of bandgap engineered laser structure for achieving ultra-broadband light sources [13, 14].

\section{ACKNOWLEDGMENT}

The work was supported by King Abdullah University of Science and Technology's Competitive Research Grant CRG-1-2012-OOI-010.

\section{REFERENCES}

[1] B. S. Ooi, H. S. Djie, Y. Wang, C. L. Tan, J. C. M. Hwang, X. M. Fang, J. M. Fastenau, A. W. K. Liu, G. T. Dang and W. H. Chang, "Quantum dashes on InP substrate for broadband emitter applications," IEEE Journal of Selected Topics in Quantum Electronics, vol. 14, pp. 1230-1238, 2008.

[2] A. Kovsh, I. Krestnikov, D. Livshits, S. Mikhrin, J. Weimert and A. Zhukov, "Quantum dot laser with 75nm broad spectrum of emission," Optics letters, vol. 32, pp. 793-795, 2007

[3] H. S. Djie, B. S. Ooi, X. M. Fang, Y. Wu, J. M. Fastenau, W. K. Liu and M. Hopkinson, "Room-temperature broadband emission of an InGaAs/GaAs quantum dots laser," Optics letters, vol. 32, pp. 44-46, 2007.

[4] H. S. Djie, C. L. Tan, B. S. Ooi, J. C. M. Hwang, X. M. Fang, Y. Wu, J. M. Fastenau, W. K. Liu, G. T. Dang and W. H. Chang, "Ultrabroad stimulated emission from quantum-dash laser", Appl. Phys. Lett., vol. 91, pp. 111116, 2007.

[5] Z. Y. Zhang, R. A. Hogg, X. Q. Lv and Z. G. Wang, "Self-assembled quantum-dot superluminescent light emitting diodes," Adv. Opt. Photon. 2(2), 201-228 (2010).

[6] P. Bardella, M. Rossetti and I. Montrosset, "Modeling of broadband chirped quantum-dot super-luminescent diodes," IEEE Journal of Selected Topics in Quantum Electronics, vol. 15, pp. 785-791, 2009.

[7] Z. Y. Zhang, Z. G. Wang, B. Xu, P. Jin, Z. Z. Sun and F. Q. Liu, "Highperformance quantum-dot superluminescent diodes," Photonics Technology Letters, IEEE, vol. 16, pp. 27-29, 2004.

[8] C. L. Tan, H. S. Djie, C. K. Tan, V. Hongpinyo, Y. H. Ding and B. S. Ooi, "The effect of multi active junctions on broadband emission from InAs/InGaAlAs quantum-dash structure," LEOS Annual Meeting Conference Proceeding (LEOS'09), pp. 147-148, 2009.

[9] M. Z. M. Khan, T. K. Ng, U. Schwingenschlogl and B. S. Ooi, "Spectral Analysis of Quantum Dash Lasers: Effect of Inhomogeneous Broadening of the Active Gain Region," IEEE Journal of Quantum Electronics, vol. 48, pp. 608-615, 2012.

[10] M. Z. M. Khan, T. K. Ng, U. Schwingenschlogl, P. Bhattacharya and B. S. Ooi, "Modeling the lasing spectra of InAs/InP quantum dash lasers," Applied Physics Letters, vol. 98, p. 101105, 2011.

[11] M. Z. M. Khan, T. K. Ng, U. Schwingenschlogl, P. Bhattacharya and B. S. Ooi., "Effect of the number of stacking layers on the characteristics of quantum-dash lasers," Optics Express, vol. 19, pp. 13378-13385, 2011.

[12] M. Z. M. Khan, T. K. Ng, and B. S. Ooi, "Influence of Vertical Coupling on the Lasing Operation of Quantum-Dash Laser," Photonics Global Conference (PGC), pp. 1-3, 2012.

[13] M. Z. M. Khan, T. K. Ng, C.-S. Lee, P. Bhattacharya and B. S. Ooi, "Chirped InAs/InP quantum-dash laser with enhanced broad spectrum of stimulated emission," Applied Physics Letters, vol. 102, p. 091102, 2013.

[14] C. Gmachl, D. L. Sivco, R. Colombelli, F. Capasso and A. Y. Cho, "Ultra-broadband semiconductor laser," Nature, vol. 415, pp. 883-887, 2002 . 